\title{
A Generalized Coverage Matrix Method for Power Quality Monitor Allocation Utilizing Genetic Algorithm
}

\author{
D. P. S. Gomes ${ }^{1}$, M. Oleskovicz ${ }^{1}$, T. R. Kempner ${ }^{1}$, and J. R. Lima Filho ${ }^{2}$ \\ ${ }^{1}$ Department of Electrical and Computer Engineering \\ University of São Paulo - Engineering School of São Carlos \\ Laboratory of Electrical Systems - Avenida Trabalhador São-carlense, 400 - São Carlos (Brazil) \\ Phone: +55 1633738149 , e-mail: douglaspsg@ usp.br, olesk@ sc.usp.br, kempner@usp.br \\ ${ }^{2}$ Eletrobrás Piauí Distribution - EDPI \\ Research Management, Development and Energy Effiency - DRRD - Av. Maranhão, 759 - Teresina, PI, Brazil \\ Phone: +55 86 3228-8010/8262, e-mail: ribamarlima@ eletrobraspiaui.com
}

\begin{abstract}
With the increase of sensitive loads and, in many cases, causing disturbances in distribution systems, power quality monitor allocation has become a subject with increasingly importance. Therefore, it becomes necessary to have well-formed strategies to allocate such equipment, since they have high cost that needs to be minimized. With this objective, this research provides a methodology for monitor's allocation that seeks to minimize the amount of equipment needed to observe voltage sags in distribution systems. The developed methodology is based on the fault voltage matrix, to obtain the residual voltage during faults, on the coverage matrix and the application of a genetic algorithm as optimization tool. The research also brings as contribution, changes in traditional methodology to better represent the indicated phenomena in distribution systems and to give better support to fault location studies, justifying a development of a generalized coverage matrix. To test this methodology, the IEEE 34-node test feeder was utilized with promising results reported and commented further.
\end{abstract}

\section{Key words}

Power Quality, Monitor Allocation, Generalized Coverage Matrix, Genetic Algorithm.

\section{Introduction}

In today's modern technological world, the availability, reliability and the Power Quality (PQ) are very important factors. The main reason for this is related to the increase of sensitive equipment and, in many instances causing disturbances present in industrial systems. Consequently, to diagnose and to continuously monitoring such phenomena becomes a major challenge for researchers and companies responsible for a good electricity supply in Distribution Systems (DS).

From all disturbances related to poor PQ, Voltage Sags (VS) are considered to be the most common type, having the most direct impacts and load damaging to final users [1]. This fact justify the need for continuously monitoring these phenomena through techniques that allow the cost minimization associated with the monitoring plan, as well as a greater sensitivity and observability of the optimally installed equipment.

In recent years, many studies have been conducted towards monitoring PQ problems in DS. To allocated monitors in order to observe VS, the Coverage Matrix (CV) has been presented and used in [2] and [3]. However, the use of this matrix is not generally suitable for radial DS. Thus, a new modification of this matrix is proposed in [4], named Topological Monitor Reach Area (TMRA), which considers the inherent topological differences between DS for monitor allocation.

Different methodologies have been also discussing the optimal monitor allocation on the PQ context. As for example, methods based on linear programming [5], that had as main goal the reduction of monitoring costs to a minimum and the maximization of the observation of monitored nodes. The application of multiobjective evolutionary algorithms was presented in [6] by Nondominated Sorting Genetic Algorithm (NSGA-II), as an elitist strategy to obtain the Pareto Frontier for the formulated problem. In [7], a method utilizing multivariable regression was introduced, where the objective was to detect similar behaviors among nodes allowing the reduction of measurement equipment, determining their optimal allocation.

Regarding optimization tools, Genetic Algorithms (GA) stand out because they are heuristics of evolutionary methods that achieve good results in a known search space. They are used in many of these different methodologies, as in [4], [8], [9] and [10].

In this context, the present paper has the monitoring of VS caused by all possible types of short circuits that may occur in DS as main goal. For such, strategies were used to allocate monitor utilizing the $\mathrm{CV}$, applying a set of modifications that allowed redefine the $\mathrm{CV}$ as a Generalized Coverage Matrix (GCM) and GA as optimization tool. To test the proposed methodology, it is worth to mention that it was used the IEEE 34-node test feeder [11], as the promising results found were reported further in section 5 of this paper. 


\section{Data acquisition and preprocessing}

To optimize the monitoring system that may face VS, one must first have access to the recorded data of the DS to be simulated. These should properly represent the real electrical system, including parameters such as lines impedances, transformers characteristics, loads, capacitor data, etc.

\section{A. Computational Modeling}

Computer simulation programs are a powerful tool in the study of the electrical systems behavior facing the different operating states that it may occur.

In this study development, it was used the software ATP (Alternative Transients Program) [12] by the graphic interface ATPDraw [13]. ATP is a universal program system for digital simulation of transient phenomena of electromagnetic as well as electromechanical nature. With this digital program, complex networks and control systems of arbitrary structure can be simulated. ATPDraw is a graphic interface, preprocessor to the version of the ATP program via MS-Windows platform.

\section{B. Fault Voltage Matrix - FVM}

As the next step, the Fault Voltage Matrix (FVM) is obtained from the simulation of different and distinct short circuits situations that the DS may face. The necessity of this analysis applying short circuits is justified, since they are the main cause of VS [9].

The FVM is a matrix that records all amplitudes of the postfault voltages observed in DS nodes, which is resulting from the short-circuit conditions applied to the DS in analysis. In its purest form, the matrix has the same number of rows and columns, where each element $\boldsymbol{T}_{(\boldsymbol{k}, \boldsymbol{f})}$ represents the magnitude of the residual post-fault voltage in the node $k$ to an applied fault in node $f$ [14].

The simulated faults for the FVM construction can assume different types (single-phase, two-phase, three-phase, with or without ground involvement), only depending on the nature of the disturbance to be analyzed.

A FVM shows how the faults propagates in the studied system according to its topology, giving also, the idea of how robust the system is versus these simulated faults, and which locations will be more or less affected by these disturbances.

Fig. 1 (a) illustrates a DS example with six nodes, where a three-phase short-circuit is applied with no fault impedance in node \#3. In Fig. 1 (b) it is showed the resulting FVM for the three-phase short-circuit simulated in each of the nodes of the DS example. It can be noticed that each column of this matrix represents the applied fault position, as in node \#3 in bold, and their rows show the registered voltages for each applied fault.

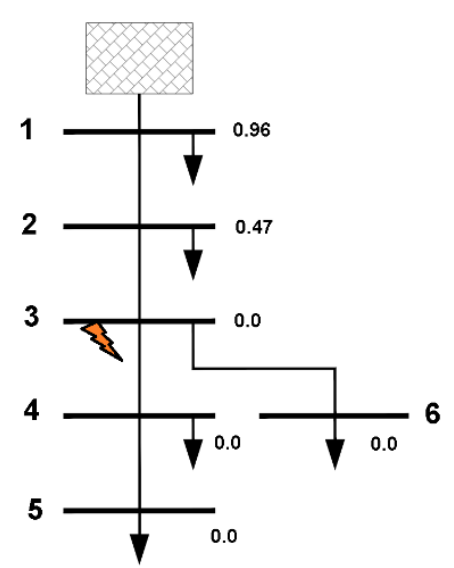

(a)

$\begin{array}{ccccccc}\text { Nodes: } & \# 1 & \# 2 & \# 3 & \# 4 & \# 5 & \# 6 \\ F V M=\left[\begin{array}{cccccc}0 & 0.82 & \mathbf{0 . 9 6} & 0.98 & 0.99 & 0.98 \\ 0 & 0 & \mathbf{0 . 4 7} & 0.65 & 0.89 & 0.66 \\ 0 & 0 & \mathbf{0} & 0.35 & 0.67 & 0.34 \\ 02 & & V 3 \\ 0 & 0 & \mathbf{0} & 0 & 0.32 & 0.28 \\ 0 & 0 & \mathbf{0} & 0 & 0 & 0.23 \\ 0 & 0 & \mathbf{0} & 0.29 & 0.61 & 0\end{array}\right] \text { V5 } \\ V 6\end{array}$

(b)

Fig. 1. (a) Radial distribution system as example. (b) Resulting FVM matrix.

\section{Coverage Matrix (CM) and Topological Coverage Matrix (TCM)}

After obtaining the FVM, a voltage threshold $(\alpha)$ was settled to indicate how low the value of the residual voltage must be to trigger (sensitize) the equipment for VS occurrences. To do so, one must set up a CM that will analyze the voltage values in the studied node for each fault position.

The CM has the same dimensions as the FVM, and it is obtained by the comparison of the values of the residual voltages with the voltage threshold, according to (1). Where, each element of the CM is replaced by 1 (one) when the magnitude of the residual voltage at the node is less or equal to the established threshold in any phase, and 0 (zero) to other cases [15].

$C M_{(j, k)}=\left\{\begin{array}{l}1, \text { if } T_{(k, f)} \leq \alpha p \cdot \text { u. in any phase } \\ 0, \text { se } T_{(k, f)}>\alpha p . u . \text { in any phase }\end{array}\right.$

Often enough, the chosen voltage threshold, $\alpha$, is arbitrated as 0.9 p.u., because it is the value determined by standard from which a VS can be characterized. VS can be described by a scenario where the effective voltage magnitude of the considered node is in the range of 0.1 to 0.9 p.u. [16]. The CM presented in the sequence (2) has the values shown in Fig. 1 (b) as reference, considering a voltage threshold of 0.9 p.u.. 


$$
C M_{0.9}=\left[\begin{array}{llllll}
1 & 1 & 0 & 0 & 0 & 0 \\
1 & 1 & 1 & 1 & 1 & 1 \\
1 & 1 & 1 & 1 & 1 & 1 \\
1 & 1 & 1 & 1 & 1 & 1 \\
1 & 1 & 1 & 1 & 1 & 1 \\
1 & 1 & 1 & 1 & 1 & 1
\end{array}\right]
$$

It is worth mentioning that, historically, the creation of this methodology has been formulated for meshed transmission systems [17]. When applied in a radial DS, a particular characteristic arises. As fact, the monitors allocation resulted from a suitable monitoring system should give enough information for VS source location tracking where a low voltage at the monitored node indicates that it is close to fault location and vice versa [14]. However, in column three of the example (Fig. 1 (b)), it can be seen that the measured voltage at nodes \#4, \#5 and \#6 suggests that the short-circuit situation was also applied at these nodes, which is not true. Such indication is influenced by the DS topology, causing zeros (0) to appear off the main diagonal of the FVM. A new proposition for the CM is presented in [4], by excluding these zeros that do not represent the VS properly. With this concept in mind, this research also suggests a new modification, which is done by not letting the downstream nodes to monitor the applied fault. This can give a better support for the fault location system and gives a greater topological differentiation of the DS. When a situation like described arises, it will be defined as a Topological Constraint. To exemplify this change in a better way, its construction is described in (3).

$$
\operatorname{TCM}_{(j, k)}=\left\{\begin{array}{c}
1, \text { se } T_{(k, f)} \leq \alpha p . u \text {.in any phase } \\
0, \text { if there is a topological constraint } \\
0, \text { se } T_{(k, f)}>\alpha p . u \text {. in any phase }
\end{array}\right.
$$

This new CM receives the name Topological Coverage Matrix (TCM). The following matrix illustrates the difference between the TCM and the CM for the example system.

$$
T C M_{0.9}=\left[\begin{array}{llllll}
1 & 1 & 0 & 0 & 0 & 0 \\
0 & 1 & 1 & 1 & 1 & 1 \\
0 & 0 & 1 & 1 & 1 & 1 \\
0 & 0 & 0 & 1 & 1 & 0 \\
0 & 0 & 0 & 0 & 1 & 0 \\
0 & 0 & 0 & 0 & 0 & 1
\end{array}\right]
$$

The TCM, as well as the CM, can be built for different voltage thresholds. In the examples shown so far (2 and 4), they were built when a threshold of 0.9 p.u. was set.

It is worth to anticipate that in this research, a new approach will be given to this threshold. The idea is to build matrices with different thresholds in order to improve the study for the possible schemes of monitor's allocations in the DS. The matrix (5) exemplifies how would be a TCM with a 0.7 p.u. as threshold.

$$
T C M_{0.7}=\left[\begin{array}{llllll}
1 & 0 & 0 & 0 & 0 & 0 \\
0 & 1 & 1 & 1 & 0 & 1 \\
0 & 0 & 1 & 1 & 1 & 1 \\
0 & 0 & 0 & 1 & 1 & 0 \\
0 & 0 & 0 & 0 & 1 & 0 \\
0 & 0 & 0 & 0 & 0 & 1
\end{array}\right]
$$

\section{Vulnerability and Affected Area}

From the TCM's rows, a Vulnerability Area can be inferred. It will indicate the sensitivity if each equipment installed on the analyzed node facing short-circuits simulated at others nodes. For example, if a monitor was installed in the node \#2, considering a TCM with a 0.9 p.u. threshold (4), it is possible to realize that it will be sensitized by faults applied at the nodes \#2, \#3, \#4, \#5 and \#6.

That said, if two monitors were installed on the nodes \#1 and \#2 each, it could be ensure that all VS that the system may face due to three-phase faults with no impedance will be observed. This is the main objective related to the problem of optimal monitor allocation, to ensure that all system nodes are observed when faults that lead to VS happen.

The observation of the VS will also be guaranteed facing three-phase faults when the monitor's allocation is set at nodes \#1 and \#3. However, in the first commented allocation scheme, faults in node \#2 are observed for both monitors installed at nodes \#1 and \#2. This repetition in observations characterizes what was named as Measurements Redundancy, and it should be maximized, because the higher this value is, the higher will be the reliability added to the monitoring system. Reliability increases from the fact that if a monitor for some reason does not record a measurement, another monitor could still be sensitized and register such situation.

The study of the Affected Area relates to the mapping of voltage sags propagation areas [18]. This region, which differs from the vulnerability area (observed through the TCM rows), is related to its columns.

For example, with a TCM built with a 0.9 p.u. voltage threshold (4), considering a fault on node \#2, two other nodes would be sensitized with VS, that is, nodes \#1 and \#2. However, if a 0.7. p.u. was set as threshold (5), the sensitized node would be only the node \#2 itself. Thus, considering lower thresholds, the affected area tends to be lower, because the VS is more severe nearest of the fault location, reducing the number of affected nodes, giving then better support to the fault location system if it is a necessary analysis [6].

\section{Proposed methodology}

In the development of this study, some differences in the proposed methodology can be clearly observed when compared to studies in the related literature as [4], [9] and [10]. Most of them relates to the construction of the matrices FVM and TCM, which are of utmost importance since it will provide the main input data to the optimization method. 


\section{A. The FVM construction}

The first point to be discussed is related to the type and fault impedance to be considered. Studies that present methodologies to optimize monitor allocation as [9] and [18], generally do not consider any value for fault impedance in their analysis. In this sense, the FVM reflects the system robustness facing simulated short-circuits and monitors are allocated in nodes with a large vulnerability area. Some other researches [10] choose to use statistical methods, such as Monte Carlo simulation, to consider all fault types that the system may face with several fault impedance values.

In this study, the selected type of short-circuit to be applied was the less severe (the one that least affects the DS), and the chosen fault impedance value $(\Omega)$ was the biggest value that causes a VS, with a $50 \Omega$ as threshold value. The reason for these choice was related to the fact that if the allocation configuration is able to guarantee the observation for these low severity phenomena, it will also be able to observe more intense disturbances. The fault impedance value is related to the same fact, because as higher this value gets, the lower the disturbance severity will be.

It should be emphasized that both the fault type (less severe) and the fault impedance (higher value), will directly impact on the construction and interpretation of the FVM.

\section{B. CM Construction}

As previously shown, the TCM can have changes in its affected area when the voltage threshold is changed. The lower the value is, the smaller will be its affect area and more efforts from the monitoring system will be required to ensure the VS observation, due to the increase of monitors. However, some systems requires a small amount of equipment to observe the VS caused by short-circuits (when a threshold of 0.9 p.u. is considered), and the utilities or companies responsible for the monitoring system could still have as objective its expansion. Such situation would increase the number of installed monitors, consequently improving the DS observability and efficiency of the implemented monitoring system. The application of different thresholds in the TCM construction can indicate good locations to allocate these exceeding monitors. In this study analysis, schemes of monitor allocation by a TCM with different threshold will be considered, and this resulting matrix will be named Generalized Coverage Matrix (GCM).

\section{Genetic Algorithm}

Genetic algorithms are generalized method for search and optimization that simulate the natural process of evolution following the Darwinian idea of natural selection. These algorithms are based on the genetic processes of biological organisms, encoding a possible solution for a "chromosome" problem composed by a string of bits and characters. These chromosomes represent individuals that are taken over several generations, in similar fashion to natural problems, evolving according to the principles of natural selection and survival of the fittest [14].

The determination of the minimum number of monitors and their optimal allocation are related problems, because the minimum number of equipment is achieved through their optimal locations, i. e., installing monitors at strategic nodes (nodes with the highest observability capacity) [14]. Thus, the GA used to formulate a solution for this problem reveals itself as a good alternative, since the problem is solved by the determination of the best solution among many others allocation possibilities.

Being the allocation problem characterized as a binary search problem, and that it does not have a high complexity, a GA in its simplest form was chosen. In this regard, the following parameters were considered:

- One point crossover;

- Selection of the best parents by the roulette wheel selection method;

- Mutation rate was set as 5\% in the initial iteration process and as the algorithm evolves the value is raised to guarantee a higher population diversity;

- Elitism to ensure the presence of the best individual found in previous interaction in the next population.

The developed algorithm have as its objective function the minimization of the number of monitors to ensure the observation of all VS caused by low severity situations of faults that the system may face. Its main constraints are related to the fact that all nodes in the DS must be observed in short-circuit situations by at least one monitor. Even so, the problem has as its secondary goal to find the solutions with the greatest measurements redundancy.

\section{Electric system analyzed}

For the proposed methodology presentation, one of the IEEE test node feeders [11] was chosen. The 34-node test feeder is a real system, located in the state of Arizona, USA. Its nominal voltage is $24.9 \mathrm{kV}$ and its main features are:

- Very long and lightly loaded (the length of the furthest node reaches $57.6 \mathrm{~km}$ );

- Two voltage regulators to maintain a good voltage profile;

- A lower transformer with $24.9 \mathrm{kV} / 4.16 \mathrm{kV}$ as nominal voltage between nodes \#25 and \#32;

- Unbalanced fix and distributed loads; and

- Shunt capacitor bank.

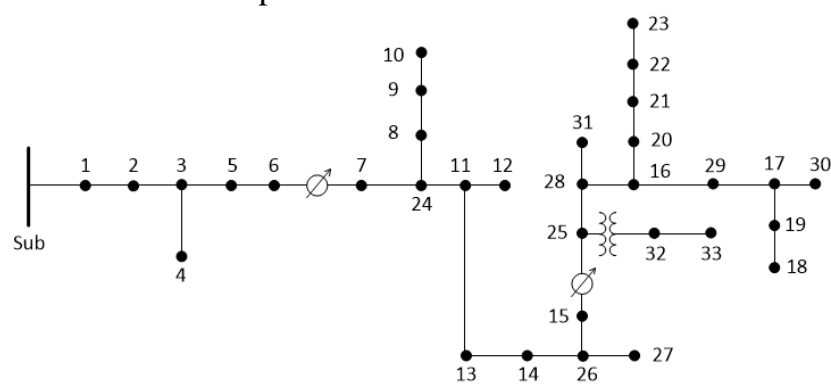

Fig. 2. IEEE 34-node test feeder single-line diagram. 


\section{Results}

After modeling of the studied system on the ATP software, several fault situations were applied to determine what type these disturbances had the less severe nature. From this analysis, the type of fault found with less severe characteristic was the single-phase related to the phase $B$, which coincided with the least loaded phase of the modeled DS.

After the type and value of fault impedances was set, simulations of this occurrence were performed in all nodes of the DS for the TCM construction. Since the matrix has a big dimension, Fig. 3 show its graphic form when a threshold of 0.9 p.u. is set. Where, one (1) values are represented by blue points and the zeros (0) by red ones, which characterize a TCM.

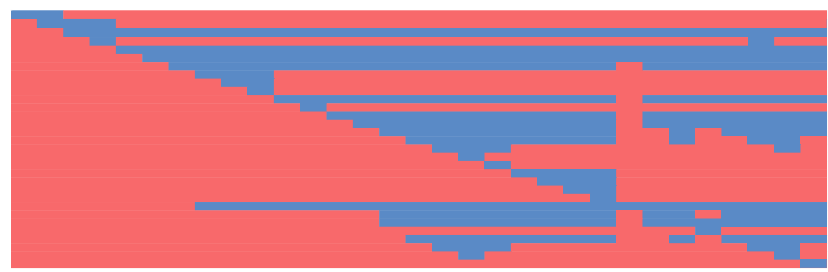

Fig. 3. Graphic illustration for the TCM with a 0.9 p.u. as threshold.

With this concept, monitors should be allocated so that all nodes are observed in VS situations (voltage magnitude less or equal to 0.9 p.u.). After the GA was executed for this configuration of TCM, the found solution was to allocated two monitors in nodes \#1 and \#3 with measurements redundancy of 2 .

Fig. 4 illustrates the monitor's allocation scheme in the IEEE 34-node test feeder, considering the distance between nodes in a representative scale to the real DS. In this figure, the green lines indicates a single-phase branch related to phase $A$, phase $B$ is in blue and the orange color represents the lowest voltage branch (system's secondary transformer).

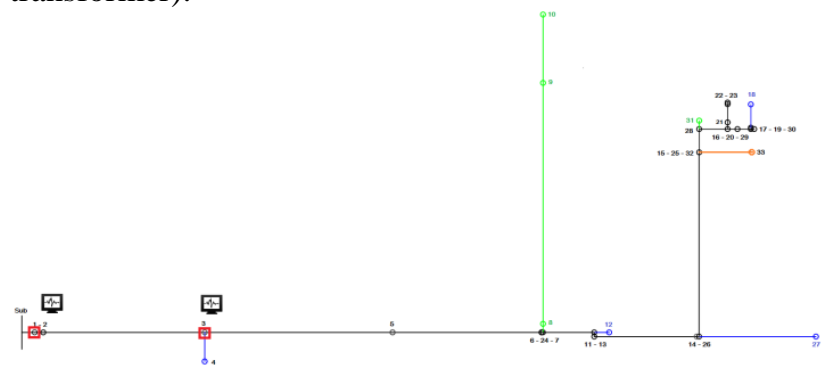

Fig. 4. Illustration for the two monitors allocation for the TCM with a threshold of 0.9 p.u..

The allocation scheme shown in Fig. 4 indicates the location where the monitors should be installed for the VS observations caused by single-phase faults related to phase $B$ (less severe) with the highest value for fault impedance (capped at $50 \Omega$ ).

However, this solution can still be refined if the company or energy utility responsible for the monitoring system wants greater measurements redundancy and more observation points. For this possibility, it was also considered in this study the decrease of the adopted threshold for the TCM construction, as it decreases the affected area and makes possible to obtain good points for the additional allocation.

Yet a problem arises when the voltage threshold is decreased for the TCM construction. If the threshold is decreased, for example to 0.8 p.u., nodes that has residual voltages between 0.8 and 0.9 p.u. can no longer be observed for the analyzed severity, since the 1 (one) value in the matrix only shows when the residual voltage is below the stipulated value.

A solution for this problem was to let the nodes (columns) with residual voltages between 0.8 and 0.9 p.u. with the same threshold and adopt a new one for the other nodes in the system. Accordingly, there is now a matrix composed with different threshold values which characterize different affected areas, thus making a GCM. The matrix then can be compiled for various voltage thresholds. This paper will present thresholds from 0.9 to 0.7 p.u. and 0.9 to 0.5 p.u..

Fig. 5 shows the GCM with 0.9 to 0.7 p.u. as thresholds. It is possible to identify a large decrease in the affected area for the simulated faults.

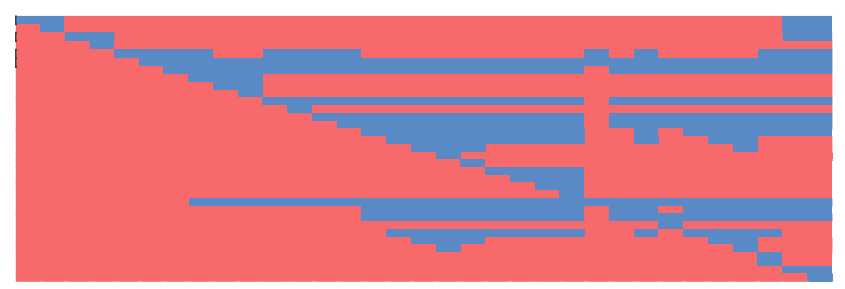

Fig. 5. Graphic illustration for the GCM with 0.9 to 0.7 p.u. as thresholds.

After the execution of the genetic algorithm for this GCM, the best solution found was to allocate monitors on the nodes \#1, \#3, \#5 and \#6, with measurements redundancy equal to 16 . Although the nodes seem to be very close in the single-line diagram (Fig. 2), Fig. 6 shows the allocation scheme in a representative scale. Through that, one can observe a good geographical spacing between the allocated monitors.

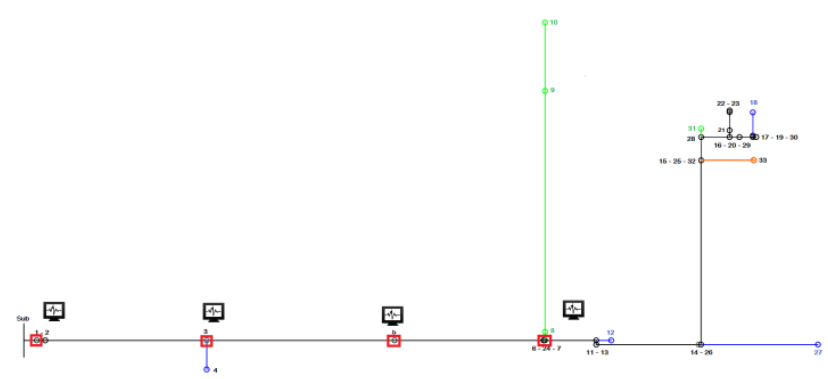

Fig. 6. Illustration of the allocation scheme for the GCM with 0.9 to 0.7 p.u. as thresholds.

The same procedure was then applied to a voltage threshold of 0.9 to 0.5 . p.u.. The result after the GA was executed is shown in Fig. 7. The best setting found was to allocate monitors at nodes \#1, \#3, \#5, \#6, \#9, \#14,\#25 and \#27, with measurement redundancy equal to 11 . 


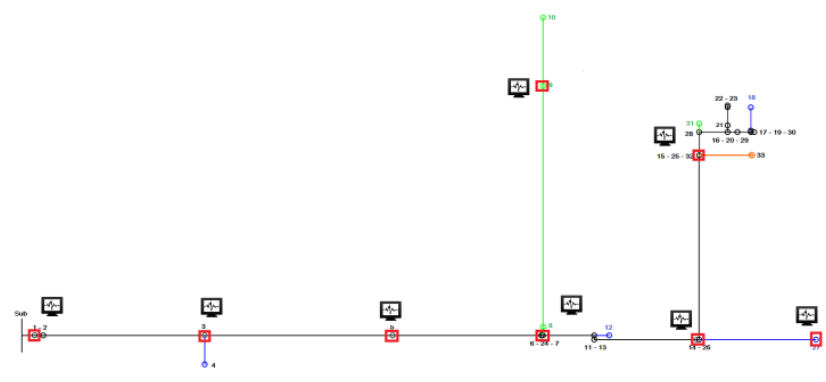

Fig. 7. Illustration of the allocation scheme for the GCM with 0.9 to 0.5 p.u.. as threshold.

It is worth noting that, despite the large amount of monitors in the last configuration and the presence of monitors in single-phase branches, the allocation of these equipment in these nodes are not required to ensure the VS observation, because just the scheme with 0.9 p.u. as threshold alone would be enough. However, by reducing the affected area and finding these locations, the professional responsible for the monitoring system can identify interesting alternatives to allocated monitors if he has more than the minimum required, or if he just wants improve even more the efficiency of the monitoring system.

\section{Conclusion}

A methodology was proposed and discussed to improve the observation of VS occurrences in an optimal way.

Through this discussion, the TCM had shown itself as a good alternative to ensure the observation of these phenomena when the type and fault impedance are chosen properly.

In addition, the GMC was presented as an interesting option to identify and point out the possible nodes on the DS to a expansion process of the initially deployed monitoring system.

The results were presented for different voltage thresholds and shown to be satisfactory, particularly with regards to support fault location systems (influenced by the TCM differentiation), from the well-spaced and defined areas (zones) of coverage.

Future studies may be responsible for not only ensure the observation of VS, but also to allow a better understanding of how the allocation scheme found relates to other aspects and problems in the context of power quality.

\section{Acknowledgements}

The authors would like to acknowledge the Department of Electrical and Computer Engineering, São Carlos School of Engineering, University of São Paulo (EESC-Brazil), for the research facilities provided to conduct this project. Our thanks also extend to the financial support received from CAPES (Coordenação de Aperfeiçoamento de Pessoal de Nível Superior), from FAPESP (Fundação de Amparo à Pesquisa do Estado de São Paulo) and Eletrobras Distribuição Piauí - EDPI for the support on the "research and development" project (PD-0042/2014-ANEEL).

\section{References}

[1] M.H.J. Bohen and I.Y.H. Gu, "Signal Processing of Power Quality Disturbances," New York: IEEE Press, 2006.

[2] G. Olguin, F. Vuinovich and M.H.J. Bollen, "An Optimal Monitoring Program for Obtaining Voltages Sag System Indexes,” IEEE Trans. Power Syst., vol. 21, pp. 378-384, 2006.

[3] M. Haghbin and E. Farjah, "Optimal Placement of Monitors in Transmission System using Fuzzy Boundaries for Voltage Sag Assessment," presented at the IEEE Power Tech Conf., Bucharest, Romania, June 28 - July 2, 2009.

[4] A. A. Ibrahim, A. Mohamed and F H. Sharee, "Optimal placement of power quality monitors in distribution systems using the topological monitor reach area," in Electric Machines \& Drives Conference (IEMDC), 2011 IEEE International, pp. 394 399, May. 2011.

[5] B. L. Liu and Q. L. Li, "The optimal allocation of power quality monitoring device," J. Proceedings of the Chinese Society of Universities for Electric Power System and Automation, vol. 21, no. 6, pp.69-73, Dec.2009.

[6] Z. N. Wei, S. Wu, G. Q. Sun, L. F. Tang and C. Wang, "Optimal placement of power quality monitors based on multiobjective evolutionary algorithm," J. Power System Technology, vol. 36, no. 1, pp. 176-181, Jan. 2012.

[7] A. Kazemi, A. Mohamed and H. Shareef, "A Novel PQM Placement Method Using Cp and Rp Statistical Indices for Power Transmission and Distribution Networks," IEEE International Power Engineering and Optimization Conference. Selangor, Malaysia, June 2011.

[8] M. Oleskovicz, H. M. G. C. Branco, R. P. M. da Silva, D.V. Coury and A. C. B. Delbem, "A Compact Genetic Algorithm Structure Used for the Optimum Allocation of Power Quality Monitors Based on Electrical Circuit Topology," In Harmonics and Quality of Power (ICHQP), 2012 IEEE 15th International Conference. Hong Kong, pp. 34 - 39, June 2012.

[9] A. A. Ibrahim, A. Mohamed, H. Shareef and S.P. Ghoshal, "Optimal Placement of Voltage Sag Monitors Based on Monitor Reach Area and Sag Severity Index," The 2010 IEEE Student Conference on Research and Development (SCOReD), Putrajaya, Malaysia, Dec. 13-14, 2010.

[10] J. C. Cebrian, C.F.M. Almeida and N. Kagan, "Genetic algorithms applied for the optimal allocation of power quality monitors in distribution networks," Harmonics and Quality of Power (ICHQP), Bergamo, 26-29 Sept. 2010.

[11] IEEE Distribution Test Feeders, "IEEE 13 Node Test Feeder", Avaliable in:

http://ewh.ieee.org/soc/pes/dsacom/testfeeders

[12] ATP - "About ATP" - Avaliable in: http://www.emtp.org.

[13] ATPDraw - "Help and about ATPDraw" - Avaliable in: http://www.atpdraw.net/help.php.

[14] C. F. M. Almeida and N. Kagan, "Allocation of Power Quality Monitors by Genetic Algorithms and Fuzzy Sets Theory," Intelligent System Applications to Power Systems, Curitiba , 8-12 Nov. 2009.

[15] H. M. G. C. Branco, "Modelagem Multiobjetivo para o Problema da Alocação de Monitores de Qualidade de Energia em Sistemas de Distribuição de Energia Elétrica," MSc. Dissertation - USP, São Carlos, pp 114, July. 2013.

[16] PRODIST. "Procedimentos de Distribuição de Energia Elétrica no Sistema Elétrico Nacional, Módulo 8 - Qualidade da Energia Elétrica," ANEEL, 2014. Avaliable in:

http://www.aneel.gov.br/area.cfm?idArea=82\&idPerfil=2.

[17] G. Olguin and M. H. J. Bollen, "Optimal Dips Monitoring Program for Characterization of Transmission System," IEEE Power Engineering Society General Meeting, 2003.

[18] T. R. Kempner, “A Robustez de um Sistema de Distribuição e a Alocação de Medidores de Qualidade de Energia Elétrica Frente aos Afundamentos de Tensão," MSc. Dissertation - USP, São Carlos, pp. 111, March 2012. 